ORIGINAL ARTICLE

\title{
Clinicopathological study of cellular proliferation and invasion in gliomatosis cerebri: important role of neural cell adhesion molecule $\mathrm{L} 1$ in tumour invasion
}

\author{
T Suzuki, S Izumoto, Y Fujimoto, M Maruno, Y Ito, T Yoshimine
}

J Clin Pathol 2005;58:166-171. doi: 10.1136/icp.2004.020909

\begin{abstract}
See end of article for authors' affiliations

Correspondence to:

Dr S Izumoto, Department of Neurosurgery, Osaka University Medical School, 2-2, Yamadaoka, Suita, Osaka, 565-0871, Japan; sizumoto@nsurg.med. osaka-u.ac.jp
\end{abstract}

Accepted for publication 31 August 2004
Background/Aims: In patients with gliomatosis cerebri (GC), glial fibrillary acidic protein (GFAP) positive cells invade the entire brain, particularly the white matter. Because the nosological definition and histogenesis of GC remain controversial, the morphology and immunohistochemical staining patterns of neoplastic GC cells were compared with those of other gliomas.

Methods: An immunohistochemical analysis of neoplastic cells from four patients with GC and 20 with astrocytic tumours using antibodies against Ki-67, GFAP, and L1, the last of which is a neural cell adhesion molecule putatively related to glioma invasion.

Results: GC tumour cells can be divided into two types, those mainly composed of strongly GFAP and L1 positive gemistocytic cells, the other composed of small, GFAP and L1 negative spindle shaped cells. The two types did not differ with respect to Ki-67 positivity. Cells from patients with other gliomas were positive for GFAP but concurrent L1 expression was negative or weakly positive.

Conclusion: The strong expression of $L 1$ in patients with $G C$ and its poor expression in the 20 patients with other types of glioma, including those with GFAP positive gemistocytic astrocytomas, suggest that L1 expression may play a role in the histogenesis of GC.
G liomatosis cerebri (GC) is a tumour of unknown origin that infiltrates the brain extensively, can involve more than two lobes, is often bilateral, and frequently extends to infratentorial structures and even the spinal cord. According to the 2000 World Health Organisation classification of tumours of the central nervous system, GC is a neuroepithelial tumour of uncertain origin whose nosological definition and histogenesis remain controversial. ${ }^{1}$ The uncertainty concerning its histogenesis is reflected in its removal from the category of undifferentiated and embryonic tumours in 1979 by the World Health Organisation ${ }^{2}$ and its inclusion, since $1993,{ }^{34}$ in the category of neuroepithelial neoplasms of unknown origin.

"We postulated that L1 plays an important role in the migration of glioma cells via homophilic binding, and that the molecule participates in the invasion of gliomas along neuronal fibres"

Pathologically, GC is characterised by diffuse enlargement of affected regions with retention of an intact configuration, proliferation of glia infiltrating the normal nervous tissue, and partial damage of nervous tissue, characterised by myelin destruction with only slight involvement of the axis cylinder and nerve cells. ${ }^{5}$ GC is composed of astrocytic cells, oligodendrocytes, transitional cells, and small undifferentiated cells exhibiting a pronounced variation in size and shape. The neoplastic cells are recognised as gliomatous by their positivity for glial fibrillary acidic protein (GFAP), although some fusiform cells are GFAP negative. The mechanism(s) underlying the tumorigenesis of GC remains to be fully elucidated, as does the composition of neoplastic cells that comprise this tumour and spread throughout intracerebral portions of the brain.

From a pathophysiological point of view, the notion that the neoplastic cells in GC exhibit the characteristics of glioma cells with respect to their origin and invasive potential is of interest. The invasiveness of glioma tumour cells is thought to be mediated by factors that include cell adhesion molecules and extracellular matrices. ${ }^{6-8}$ Ll molecules (CD171, Ll cell adhesion molecule, neural cell adhesion molecule L1), $200 \mathrm{kDa}$ glycoproteins, play an important role not only in neurone-neurone adhesion but also in neurite fasciculation, neurite outgrowth on Schwann cells, and neural cell migration. ${ }^{9-11}$ Although it is designated a neural recognition molecule, $\mathrm{Ll}$ is also expressed by certain epithelial cells and by some cells of lymphoid and myelomonocytic origin. Miura et al showed that, in co-cultures, L1 transfected fibroblasts strongly promoted the migration of rat cerebellar neural cells. ${ }^{10}$

We previously reported that $\mathrm{Ll}$ is a stimulatory factor for glioma cell and neural cell migration. Using the reverse transcriptase-polymerase chain reaction assay and fluorescence activated cell sorter analysis, we demonstrated that in culture glioma cells expressed the Ll gene, and that they are stimulated to migrate by soluble Ll molecules released from Ll transfected fibroblasts. ${ }^{12}$ In in vivo experiments, glioma cells implanted into rat brains migrated strongly towards Ll molecules injected into the contralateral cerebral hemisphere. ${ }^{13}$ However, immunohistochemical analysis revealed weak expression of $\mathrm{Ll}$ in human glioma tissue specimens. ${ }^{14}$ We postulated that $\mathrm{Ll}$ plays an important role in the migration of glioma cells via homophilic binding, and that the molecule participates in the invasion of gliomas along neuronal fibres.

To test this hypothesis in patients with GC, we subjected neoplastic cells to immunohistochemical analysis to assess cell proliferation and invasion, and to determine the involvement of Ll molecules.

Abbreviations: GC, gliomatosis cerebri; GFAP, glial fibrillary acidic protein; $\mathrm{MRI}$, magnetic resonance imaging 
Table 1 Patient details, histology, and immunohistochemical results

\begin{tabular}{|c|c|c|c|c|c|c|}
\hline Case & Age & Sex & Histology & GFAP & Ll & Ki-67 (\%) \\
\hline 1 & 47 & $\mathrm{~F}$ & Gliomatosis cerebri (As-grade 3) & ++ & ++ & 4 \\
\hline 2 & 58 & M & Gliomatosis cerebri (As-grade 3) & ++ & ++ & 17 \\
\hline 3 & 31 & M & Gliomatosis cerebri (As-grade 3) & ++ & ++ & 11 \\
\hline 4 & 45 & $\mathrm{~F}$ & Gliomatosis cerebri (As-grade 2) & ++ & ++ & 5 \\
\hline 5 & 67 & $\mathrm{~F}$ & Pilocytic astrocyłoma & ++ & + & 4 \\
\hline 6 & 20 & M & Fibrillary astrocytoma & ++ & + & 2 \\
\hline 7 & 52 & M & Fibrillary astrocytoma & + & \pm & 2 \\
\hline 8 & 52 & M & Fibrillary astrocytoma & ++ & \pm & 4 \\
\hline 9 & 43 & $\mathrm{~F}$ & Gemistocytic astrocytoma & ++ & \pm & 3 \\
\hline 10 & 52 & $\mathrm{~F}$ & Gemistocytic astrocytoma & ++ & + & 2 \\
\hline 11 & 25 & M & Oligoastrocytoma & \pm & \pm & 8 \\
\hline 12 & 31 & M & Oligoastrocytoma & \pm & \pm & 1 \\
\hline 13 & 40 & M & Oligoastrocytoma & \pm & - & 2 \\
\hline 14 & 40 & $\mathrm{~F}$ & Oligoastrocytoma & \pm & \pm & 5 \\
\hline 15 & 26 & $\mathrm{~F}$ & Anaplastic astrocytoma & ++ & + & 8 \\
\hline 16 & 54 & M & Anaplastic astrocytoma & ++ & - & 5 \\
\hline 17 & 50 & M & Anaplastic oligoastrocytoma & + & - & 19 \\
\hline 18 & 27 & M & Glioblastoma & + & \pm & 20 \\
\hline 19 & 48 & $\mathrm{~F}$ & Glioblastoma & \pm & - & 11 \\
\hline 20 & 59 & $M$ & Glioblastoma & + & \pm & 12 \\
\hline 21 & 62 & M & Glioblastoma & + & - & 8 \\
\hline 22 & 63 & $\mathrm{~F}$ & Glioblastoma & + & - & 9 \\
\hline 23 & 42 & $\mathrm{~F}$ & Glioblastoma & \pm & \pm & 12 \\
\hline 24 & 58 & $M$ & Glioblastoma & \pm & \pm & 8 \\
\hline
\end{tabular}

Immunohistochemical analysis of 4 patients with gliomatosis cerebri and 20 patients with other gliomas.

As, astrocytoma; GFAP, glial fibrillary acidic protein; -, negative; \pm , trace/positive; + , positive; ++ , strongly positive.

\section{CLINICAL MATERIALS AND METHODS Patients}

Our study population consisted of four patients with GC and 20 patients with other gliomas (one pilocytic astrocytoma, three fibrillary astrocytomas, two gemistocytic astrocytomas, four oligoastrocytomas, two anaplastic astrocytomas, one anaplastic oligoastrocytoma, and seven glioblastomas).

Detailed premortem and postmortem evaluations were performed in one patient with GC (case 1), a 47 year old woman. On Tl weighted magnetic resonance imaging (MRI), we noted areas of isointensity and hypointensity compared with the normal brain. T2 weighted MRI obtained in the terminal stage demonstrated diffuse areas of high intensity throughout the brain, especially the white matter. This patient died 18 months after being diagnosed with grade 2 astrocytoma based on histopathological examination of surgical specimens. Immunohistochemical analysis was performed on postmortem samples obtained from 24 regions. These were the bilateral frontal, temporal, parietal, and occipital lobes, the bilateral basal ganglia, caudate nucleus, thalamus, and cerebellum, the corpus callosum, midbrain, pituitary gland, upper and lower pons, the medulla oblongata, and the upper and middle cervical spinal cord. We used surgical specimens for the analysis of the other three GCs and all gliomas (table 1).

\section{Histopathology}

Tissue samples were fixed in 10\% neutral buffered formalin and $6 \mu \mathrm{m}$ thick paraffin wax embedded sections were stained with haematoxylin and eosin. For immunohistochemical analysis we used a mouse monoclonal antibody against human GFAP (diluted 1/60; Boehringer Mannheim Biochemica, Mannheim, Germany), a mouse monoclonal antibody against human Ki-67 (clone MIB-1; diluted 1/50; Dako, Carpinteria, California, USA), and a rabbit polyclonal antibody against rat $\mathrm{Ll}$ that recognises the intracellular, membrane binding, and extracellular domains of Ll. This last antibody was a gift from Dr Lemmon, Case Western Reserve University, Ohio, USA. To enhance Ki-67 immunostaining, the sections were pretreated in a microwave in $0.01 \mathrm{M}$ citrate buffer, pH 6.
After washing with Tris buffer, the sections were reacted with biotinylated antimouse IgG for GFAP and Ki-67, or antirabbit IgG for Ll (diluted 1/200; Vector Laboratories, Burlingame, California, USA). After another wash, they were reacted for 40 minutes with avidin-biotin peroxidase complex (1/50 dilution; Vectastain Elite ABC kit; Vector). For visualisation of immune complexes, sections were immersed in $0.02 \% 3,3^{\prime}$-diaminobenzidine tetrahydrochloride (DAB Wako Pure Chemical Industries Ltd, Osaka, Japan), and $0.01 \% \mathrm{H}_{2} \mathrm{O}_{2}$ in Tris buffer. Finally, slides were counterstained with haematoxylin and embedded in a universal mount. All slides were examined under a light microscope. Each slide was graded for the degree of Ll and GFAP staining, where $(-)$ indicates no staining, and $( \pm),(+)$, and $(++)$ indicate trace, positive, and strongly positive staining, respectively, and the correlation between the diagnosis and the degree of Ll staining was examined. The number of Ki-67 positive cells in 500 cells at high power ( $\times 400$ magnification) was counted in five random fields and the average number of labelled cells was reported as the Ki-67 labelling index (\%). Because it was not possible to count the exact number of infiltrating tumour cells among the normal brain cells, we compared Ki-67 labelling in tumours by averaging the positively stained nuclei in the total number of cells in the specimen.

\section{RESULTS}

In our representative case of GC (case 1), T2 weighted MRI showed diffuse high intensity regions throughout the brain, especially the white matter (fig 1). In the postmortem specimen there was infiltration by tumour cells of the entire brain (fig 1, circles); no tumour cells were detected in the lower pons and spinal cord (fig l, squares). The area stained for tumour cells was much larger than that detected by MRI. Samples from the 24 areas identified in fig 1 by squares and circles were subjected to immunohistochemistry using anti$\mathrm{Ki}-67$, anti-GFAP, and anti-Ll antibodies. Ll is a neural cell adhesion molecule that is thought to play a role in glioma cell invasion. Our findings indicated that the tumour cells could be divided into two types (fig 2); type A consists primarily of GFAP and Ll positive gemistocytic cells and type B consists of small GFAP and Ll negative spindle shaped cells. Type A gemistocytic cells were scattered throughout the entire brain 


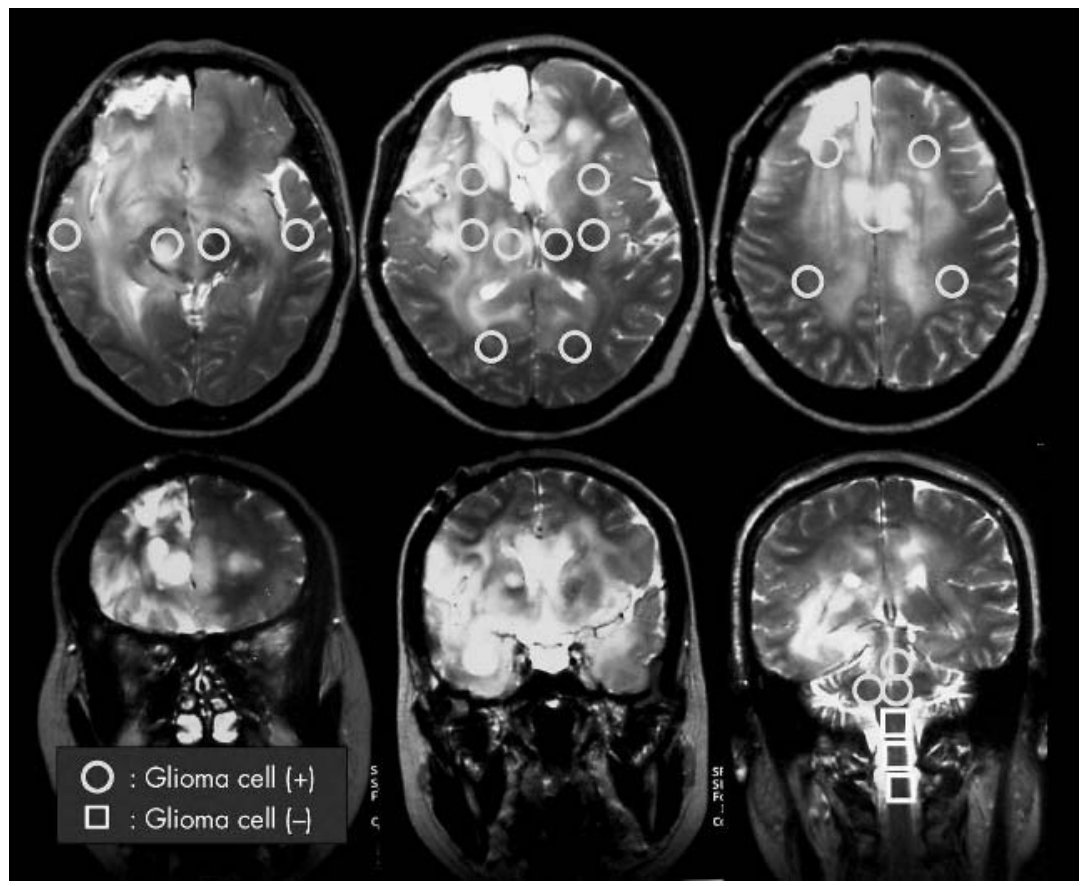

Figure 1 Neuroimages obtained during the terminal stage of patient 1. T2 weighted magnetic resonance imaging shows diffuse high intensity regions in the brain, especially the white matter. Postmortem specimens revealed tumour cell infiltration throughout the entire brain (circles) except for the lower pons and spinal cord (squares).

and their cytomembrane and cytoplasm were mainly positive for GFAP and Ll (fig 2A-C). In contrast, in type B, GFAP and Ll reactivity was weak or negative (fig $2 \mathrm{D}-\mathrm{F}$ ).

The presence of these cells in all examined brain lesions suggests that they share the same origin and are the result of local malignant transformation followed by migration throughout the entire brain. Careful assessment of Ll positive cells distributed throughout the brain revealed that not only GFAP positive gemistocytic cells were positive for Ll, but that some neurone cells were also positive. With respect to cell proliferation rates, the Ki-67 labelling index in all examined brain lesions of case 1 was $2-4 \%$ (table 1$)$. Analysis of surgical specimens from the other three GC cases revealed that tumour cells that were positive for GFAP were also strongly positive for Ll. The Ki-67 labelling index varied among these patients and did not correlate with Ll staining.

Next we compared the four patients with GC with the 20 patients with other gliomas for the expression of $\mathrm{Ll}$ and GFAP in the tumour specimens. All of our four cases of GC are primary "true GC" because they do not have distinct neoplastic masses on the image. Secondary GC is a progressive type of malignant glioma with a mass lesion as reported Ross et al. ${ }^{15}$ As shown in table 1, although Ll was strongly expressed by neoplastic cells in all patients with GC, 10 of the other 20 patients manifested only trace/positive staining and none of the samples was strongly positive for Ll. Figure 3 shows representative cases stained with anti-GFAP and anti-Ll antibodies. The degree of Ll staining did not reflect the malignancy grade of the 20 gliomas, although $\mathrm{Ll}$ positive tumours tended to exhibit invasive tendencies on MRI. In fact, in contrast to GC, none of the strongly GFAP positive glioma cells from seven of the 20 patients with other gliomas manifested strong Ll expression. Furthermore, to establish the expression of $\mathrm{Ll}$ on reactive astrocytes in non-neoplastic conditions, gliosis brain tissue caused by arteriovenous malformation haemorrhage was prepared and tissue sections were immunostained with GFAP and Ll. GFAP immunostaining was found to be positive in these samples but Ll immunostaining was trace/positive on reactive astrocytes (data not shown). These results indicate that strong Ll positivity is characteristic of GC.

\section{DISCUSSION}

GC is a diffuse tumour that infiltrates the brain extensively, involves more than two lobes, is often bilateral, and frequently extends to infratentorial structures. ${ }^{1}$ Histologically, it is composed of elongated glial cells that typically resemble astrocytes. Upon infiltration of myelinated tracts, the cells often form parallel rows between nerve fibres and exhibit a local histoarchitecture. We categorised the neoplastic GC cells as type A (GFAP and Ll positive gemistocytic cells) and type B (small, GFAP and Ll negative or weakly positive, spindle shaped cells). We postulated that cells that infiltrated the myelin fibres would be of type A, in which GFAP and Ll positive gemistocytic cells are abundant.

Among the molecules that participate in cell migration, Ll is thought to promote neuronal cell migration most strongly. ${ }^{16}{ }^{17}$ We previously demonstrated the biological importance of Ll in glioma invasion along neuronal fibres. ${ }^{13}$ Sequence analysis revealed that $\mathrm{Ll}$ expressed in the gliomas that we tested was short-type Ll (Llcs), generated by alternative splicing to delete one of the putative phosphorylation sites, ${ }^{13}{ }^{18}$ and that $\mathrm{Ll}$ mediated interactions between glioma and other cells can occur via the Llcs isoform on glioma cells.

"Our results suggest that L1 expressed on neoplastic gliomatosis cerebri cells may contribute to their invasive potential mediated by homophilic interactions with other L1 molecules present on neurones or surrounding oligodendrocytes"

A postmortem study of case 1 identified neoplastic cells throughout the bilateral cerebral white matter, with spreading to the pons; however, there were no tumour cells in the 

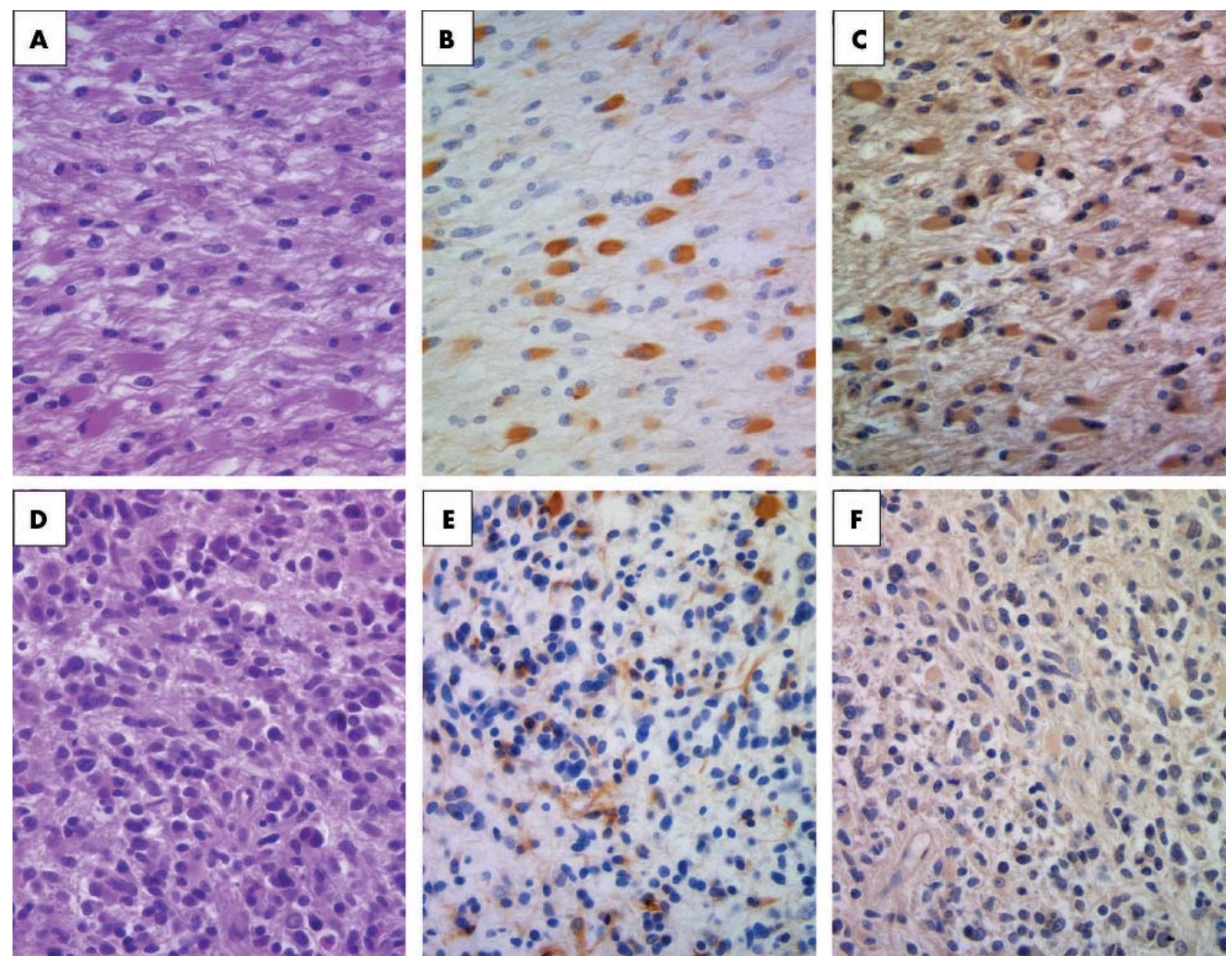

Figure 2 Postmortem specimens from case 1 revealing the morphological and immunohistochemical features of the two types of tumour cells. (A-C) Type A cells and (D-F) type B cells. (A) Haematoxylin and eosin staining of type A tumour cells shows the proliferation of gemistocytic cells. (B) Glial fibrillary acidic protein (GFAP) staining of type $A$ cells reveals positive staining of gemistocytic cell bodies. (C) L1 staining of type $A$ cells shows staining of gemistocytic cell bodies. (D) Haematoxylin and eosin staining of type B tumour cells shows the accumulation of cells with small round nuclei. (E) GFAP staining of type B cells shows faintly stained cells. (F) LI staining of type B cells shows faintly stained cells.

lower pons. This suggests that some mechanism(s) may interfere with the invasion of neoplastic GC cells from the pons to the lower structures. ${ }^{19}$ Based on their morphological and immunohistochemical features, we divided GC tumour cells into type A and B cells, both of which were scattered throughout the brain of patient 1 . Because of similarities in the configuration of these cells and their expression of Ll, we postulate the existence of one tumorigenic origin. We propose that these neoplastic cells are the result of local malignant transformation and that their diffuse spread is attributable to active migration in which Ll plays an important role. Because the neoplastic GFAP positive gemistocytic cells coexpressed Ll, we suggest that these glia derived cells proliferated and subsequently invaded the whole brain.

In our earlier study, we used frozen sections to investigate the expression of Ll by gliomas. ${ }^{14}$ In our current study, we prepared paraffin wax embedded sections from surgical specimens and found that some of the non-GC tumours exhibited weak positivity for Ll. In contrast, we found strong expression of both GFAP and Ll by the neoplastic cells in all four patients with GC. Our observation that in case 1, type A gemistocytic cells manifested strong expression of both GFAP and Ll suggests that their ability to invade the brain, and especially the white matter, was enhanced. In contrast, in two gemistocytic astrocytomas, gemistocytic cells manifested strong GFAP expression but weak Ll expression. Based on this observation, we suggest that the strong expression of Ll by migrated GC cells reflects their invasive characteristic.

Our results suggest that Ll expressed on neoplastic GC cells may contribute to their invasive potential mediated by homophilic interactions with other Ll molecules present on neurones or surrounding oligodendrocytes. Soluble L1 secreted by neural cell components around the tumour may also contribute to their migration. GC cells per se may release a soluble form of $\mathrm{Ll}$, and this may stimulate their motility and accelerate their migration far from the site of their origin via binding to $\mathrm{Ll}$, which is expressed constitutively on neuronal fibres.

Mechtersheimer et al and Fogel et al reported that Ll is expressed by many human carcinomas in addition to GC, such as uterine and ovarian cancers, and soluble $\mathrm{Ll}$ is specifically detected in serum samples from patients with these tumours. ${ }^{20}{ }^{21}$ They propose that ectodomain released Ll promotes migration by autocrine/paracrine stimulation via $\alpha_{V} \beta_{5}$ integrin. Both Ll-Ll homophilic and Ll- $\alpha_{V} \beta_{3}$ interactions play an important role in the transendothelial migration of melanoma cells. ${ }^{22}$ Recently, a novel translational mechanism to account for the association between $\mathrm{Ll}$ expression and motile processes involved in metastasis and development was provided..$^{23}$ 

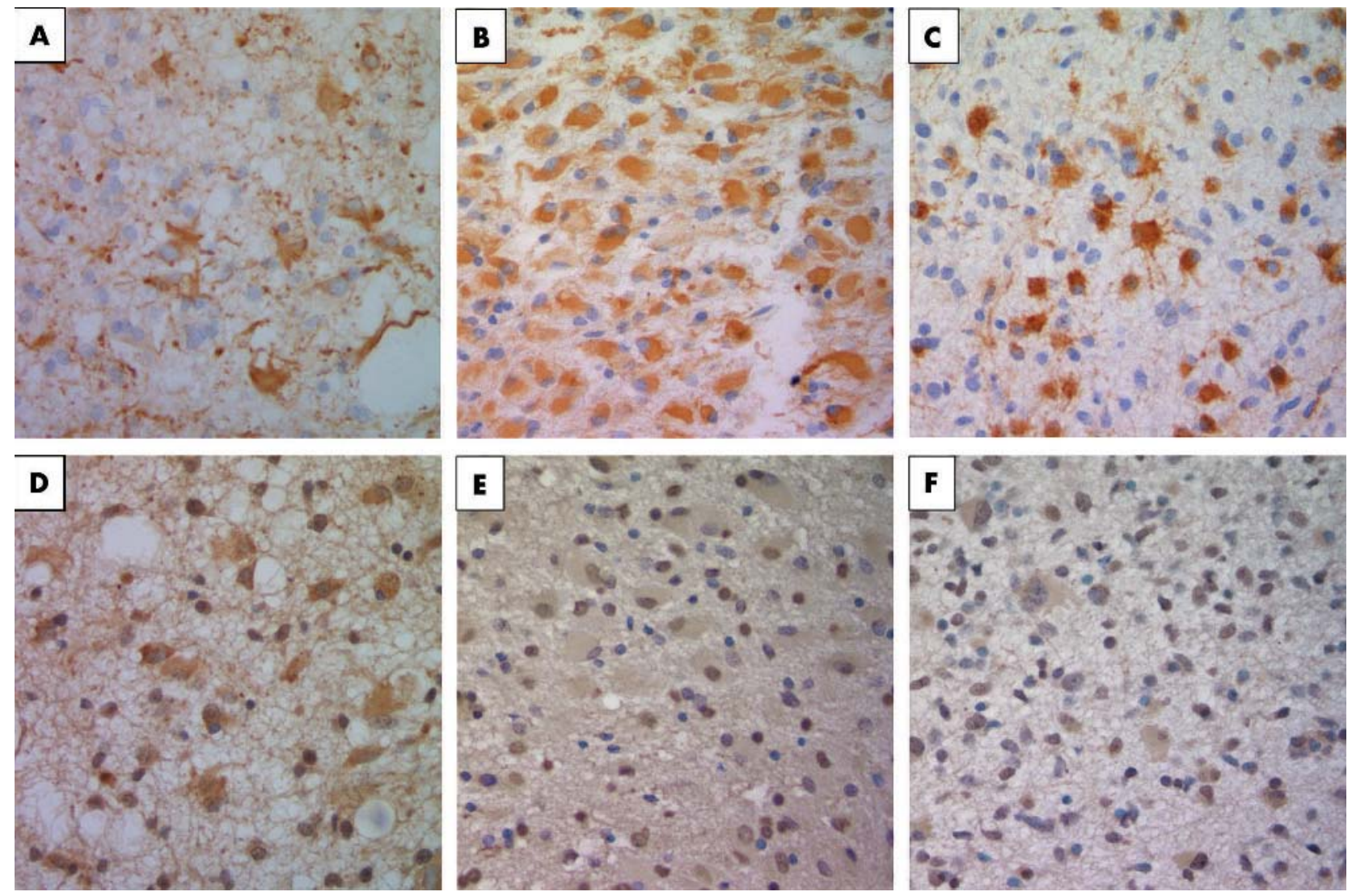

Figure 3 (A, D) Results of staining in case 2, which was representative of patients with gliomatosis cerebri. Gemistocytic cells were positive for (A) glial fibrillary acidic protein (GFAP) and (D) L1. (B, E) Results of staining in case 9, which was representative of patients with gemistcytic astrocytoma. Cells in the gemistocytic astrocytoma were (B) positive for GFAP but (E) were only weakly positive for L1. (C, F) Results of staining in case 15, which was representative of patients with anaplastic astrocytoma. Cells from anaplastic astrocytoma were (C) positive for GFAP but (F) weakly positive for L1.

Our finding that Ll is highly expressed by neoplastic GC cells, whereas other gliomas expressed Ll weakly, leads us to suggest that Ll plays an important role in the spread of GC and other cancers. The level of Ll expression did not reflect the cellular proliferative ability, but rather was related to the invasive potential of GC. Inhibiting the function of $\mathrm{Ll}$ on neoplastic GC cells may represent a breakthrough in the treatment of patients with GC. The accumulation of more clinical and neuropathological data on patients with glioma and GC will shed light on the mechanism(s) that underlie the high invasive potential of glioma cells, a major problem in the management of patients with malignant glioma and GC.

\section{Take home messages}

- Patients with gliomatosis cerebri (GC) strongly expressed glial fibrillary acidic protein (GFAP) and neural cell adhesion molecule L1, whereas patients with other types of glioma, including those with GFAP positive gemistocytic astrocytomas, expressed L1 only weakly

- These results suggest that L1 expression may play a role in the spread of GC

- Inhibiting the function of L1 on neoplastic GC cells may represent a therapeutic target in patients with GC

\section{Authors' affiliations}

T Suzuki, S Izumoto, Y Fujimoto, M Maruno, T Yoshimine, Department of Neurosurgery, Osaka University Medical School, 2-2, Yamadaoka, Suita, Osaka, 565-0871, Japan

Y Ito, Department of Clinical Pathology, Mino City Hospital, 5-7-1 Kayano, Mino, Osaka, 562-0014, Japan

\section{REFERENCES}

Kleihues P, Cavenee WK. Pathology and genetics of tumours of the nervous system. Lyon: IARC Press, 2000

2 Zulch KJ. Histological typing of tumours of the central nervous system Geneva: World Health Organisation (WHO), 1979.

3 Kleihues P, Burger PC, Scheithauer BW. The new WHO classification of brain tumours. Brain Pathol 1993;3:255-68.

4 Kleihues P, Burger PC, Scheithaver BW. Histological typing of tumours of the central nervous system, 2nd ed. Berlin Heiderberg: Springer Verlag, 1993.

5 Scheinker IM, Evans JP. Diffuse cerebral gliomatosis. J Neuropathol Exp Neurol 1943;2:178-89.

6 Noble M, Albrechtsen M, Moller C, et al. Glial cells express N-CAM/D2CAM-like polypeptides in vitro. Nature 1985;316:725-8.

7 Poltorak M, Sadoul R, Keilhaver G, et al. Myelin-associated glycoprotein, a member of the L2/HNK-1 family of neural cell adhesion molecules, is involved in neuron-oligodendrocyte and oligodendrocyte-oligodendrocyte interaction. J Cell Biol 1987; 105:1893-9.

8 Takeichi $M$. The cadherins: cell-cell adhesion molecules controlling animal morphogenesis. Development 1988;102:639-55.

9 Appel F, Holm J, Conscience JF, et al. Several extracellular domains of the neural cell adhesion molecule L1 are involved in neurite outgrowth and cell body adhesion. J Neurosci 1993;13:4764-75.

10 Miura M, Asou $H$, Kobayashi M, et al. Functional expression of a full-length cDNA coding for rat neural cell adhesion molecule L1 mediates homophilic intercellular adhesion and migration of cerebellar neurons. J Biol Chem 1992;267:10752-8.

11 Lindner J, Rathjen FG, Schachner M. L1 mono- and polyclonal antibodies modify cell migration in early postnatal mouse cerebellum. Nature 1983;305:427-30.

12 Ohnishi T, Matsumura $H$, Izumoto $S$, et al. A novel model of glioma cell invasion using organotypic brain slice culture. Cancer Res 1998;58:2935-40. 
13 Izumoto S Ohnishi T, Arita N, et al. Gene expression of neural cell adhesion molecule L1 in malignant gliomas and biological significance of L1 in glioma invasion. Cancer Res 1996;56:1440-4

14 Tsuzuki T, Izumoto S, Ohnishi T, et al. Neural cell adhesion molecule L1 in gliomas: correlation with TGF-beta and p53. J Clin Pathol 1998:51:13-17.

15 Ross IB, Robitaille Y, Villemure J-G, et al. Diagnosis and management of gliomatosis cerebri: recent trends. Surg Neurol 1991;36:431-40.

16 Asou H, Miura M, Kobayashi M, et al. The cell adhesion molecule L1 has a specific role in neural cell migration. Neuroreport 1992;3:481-4.

17 Asou H, Miura M, Kobayashi M, et al. Cell adhesion molecule L1 guides cell migration in primary reaggregation cultures of mouse cerebellar cells. Neurosci Lett 1992;144:221-4.

18 Kozma R, Ahmed S, Best A, et al. The Ras-related protein $\mathrm{Cdc} 42 \mathrm{Hs}$ and bradykinin promote formation of peripheral actin microspikes and filopodia in Swiss 3T3 fibroblasts. Mol Cell Biol 1995; 15:1942-52.
19 Artigas J Cervos-Navarro J, Iglesias JR, et al. Gliomatosis cerebri: clinical and histological findings. Clin Neuropathol 1985;4:135-48.

20 Mechtersheimer S, Gutwein P, Agmon-Levin N, et al. Ectodomain shedding of L1 adhesion molecule promotes cell migration by autocrine binding to integrins. J Cell Biol 2001;155:661-73.

21 Fogel M, Gutwein P, Mechtersheimer S, et al. L1 expression as a predictor of progression and survival in patients with uterine and ovarian carcinomas. Lancet 2003;362:869-75.

22 Voura EB, Ramjeesingh RA, Montgomery AMP, et al. Involvement of integrin $\alpha_{\mathrm{v}} \beta_{3}$ and cell adhesion molecule $\mathrm{L} 1$ in transendothelial migration of melanoma cells. Mol Biol Cell 2001;12:2699-710.

23 Silletti S, Yebra M, Perez B, et al. Extracellular signal-regulated kinase (ERK)-dependent gene expression contributes to L1 cell adhesion molecule-dependent motility and invasion. J Biol Chem 2004;279:28880-8. 